\title{
ATIVIDADE DOS HERBICIDAS FLUMIOXAZIN E METRIBUZIN EM DIFERENTES SOLOS ${ }^{1,2}$
}

\author{
MAURÍlIO F. de OLIVEIRA ${ }^{3}$, ANTÔNIO A. da SILVA ${ }^{4}$, FRANCISCO A. FERREIRA ${ }^{4}$, JORGE G. MAGALHÃES ${ }^{5}$
}

\section{RESUMO}

A atividade dos herbicidas flumioxazin e metribuzin foi avaliada, em casa-de-vegetação. em quatro tipos de solos usando areia lavada como controle. Os solos. coletados em diferentes regiões agrícolas, foram classificados como: PVc coletado em Viçosa-MG, LRd coletado em Dourados-MS, LVh em Realeza-MG e LVm em Três MariasMG. Em vasos com capaciade de $430 \mathrm{~cm}$ foram avaliadas, para cada tipo de solo, por meio de bioensaio com pepino, dez doses de cada herbicida. com cinco repetições. O nivel de umidade nos vasos foi mantido constante, próximo à capacidade de campo. durante todo o ensaio. As doses aplicadas e a quantidade de solo nos vasos variaram em função do tipo de solo. O tipo de solo, suas características fisico-químicas. especialmente $\mathrm{o}$ teor de carbono orgânico. influenciaram acentuadamente a atividade dos herbicidas. Para o flumioxazin, solos $\mathrm{cm}$ maiores teores de carbono orgânico produziram maiores valores de $\mathrm{I}_{50}$ ou seja, menor atividade do herbicida. A atividade do metribuzin foi influenciada pelo $\mathrm{pH}$ e pelo teor de carbono orgânico, nos diferentes solos. Os resultados sugerem que os herbicidas devam ser recomendados em doses diferenciadas, segundo tipo de solo.

Palavras chave: adsorção. bioensaio. carbono orgânico.

\section{ABSTRACT \\ Activity of flumioxazin and metribuzin herbicides in different soils}

The activity of flumioxazin and metribuzin herbicides was evaluated in four different soils using washed sand. The soils were: an Utisol collected in an agricultural region in the State of Minas Gerais and an Oxisol collected in three different agricultural regions; two in the State of Minas Gerais and one in the State of Mato Grosso do Sul. Ten rates of each herbicide were evaluated for each soil in pots. with five replications per treatment. Herbicide rates tested and the amount of soil per pot varied with the type of soil being evaluated. The soil moisture content was maintained at soil moisture capacity with simulated rainfalls. The soil $t^{y}$ pe, its physical-chemical properties, and mainly its organic carbon content affected the activit ${ }^{\mathrm{y}}$ rate of both herbicides. For flumioxazin, the soils with the highest organic carbon content presented the highest values of $\mathrm{I}_{50}$ which means, greater adsorption of the herbicide. Meanwhile, for metribuzin, the activity rate was affected by the $\mathrm{pH}$ and the organic carbon content of the different soils tested. The results of this study suggest that the recomendations for use of herbicides should vary according to the type of soil. carbon.

1 Recebido para publicação em 08/09/96 e na forma revisada em 09/12/97.

2 Parte da dissertação de mestrado do primeiro autor à Universidade Federal de Viçosa (UFV).

'3 Pós-Graduando, MS, Dept. Fitotecnia, UFV, Viçosa/MG, CEP: 36571-000.

4 Prof. Dept. Fitotecnia, UFV, Viçosa/MG, CEP: 36571-000.

5 Téc. Nível Superior, Dept. Fitotecnia, UFV, Viçosa/MG, CEP: 36571-000. 


\section{INTRODUÇÃO}

O destino final de todos dos herbicidas, sejam eles aplicados em pré ou em pós-emergência, é sempre o solo. A disponibilidade destes produtos para as plantas e para a biota é influenciada pelas frações orgânica e mineral do solo. Em contato com os colóides do solo, os herbicidas estão sujeitos à adsorção, lixiviação e degradação, mediante processos fisico-químicos e biológicos (Bailey \& White, 1970).

A adsorção é o fator mais importante pelo qual os herbicidas se tornam não-disponíveis para serem absorvidos por plantas e microrganismos. Todos os herbicidas são, em algum grau, adsorvidos, e sua atividade é reduzida na proporção direta da quantidade adsorvida. As moléculas adsorvidas ficam não-disponíveis para os processos biológicos, físicos e químicos até que ocorra algum processo de dessorção. A adsorção é, assim, um processo-chave na bioatividade dos herbicidas e, esta, pode ocorrer por meio de ligações hidrofóbicas, pontes de hidrogênio, forças de van der Waals, ligações metálicas e eletrostáticas (Roowell, 1994), dependendo do tipo de colóide.

O grau de adsorção pode ser influenciado pela solubilidade da molécula do herbicida havendo relação inversa entre estes dois fatores (Graveel \& Turco, 1994; Roowell, 1994). A solubilidade depende também, parcialmente. da polaridade, das propriedades elétricas do produto e do comportamento intrínseco da molécula no ambiente. Embora a solubilidade e o comportamento do produto na água sejam de importância fundamental no impacto ambiental, a recomendação de doses de herbicidas para o eficiente controle de plantas daninhas é feito em função da textura e do teor de matéria orgânica do solo (Stevenson, 1982; Roowell, 1994). Entretanto, o conhecimento da capacidade adsortiva de um herbicida, em função do tipo de solo, é importante para a definição das doses a serem utilizadas, pois pode influenciar a lixiviação, a volatilização e a decomposição microbiana (Bailey \& White, 1970).
Diversos trabalhos têm demonstrado que o $\mathrm{pH}$ e os teores de carbono orgânico e de argila do solo, influenciam a adsorção do metribuzin (Ladlie et al. 1976; Sharom \& Stephenson, 1976; Peter \& Weber, 1985). No entanto, estes trabalhos foram realizados em solos americanos, onde há predominância de argila 2:1 e as condições climáticas são bem diferentes das observadas no Brasil. Não foi encontrado na literatura nacional nenhum trabalho referente à atividade de metribuzin e flumioxazin.

O presente trabalho teve como objetivo avaliar a atividade dos herbicidas flumioxazin e metribuzin, em solos com diferentes características fisico-químicas.

\section{MATERIAL E MÉTODOS}

O experimento foi conduzido em casa-devegetação, no campus da Universidade Federal de Viçosa - MG. Foram utilizados cinco substratos, sendo quatro provenientes dos solos Podzólico Vermelho-Amarelo câmbico fase terraço $(\mathrm{PVc})$ de Viçosa - MG, Latossolo Roxo distrófico (LRd) de Dourados - MS, Latossolo Vermelho-Amarelo textura média $(\mathrm{LVm})$ de Três Marias - MG e Latossolo Vermelho-Amarelo húmico (LVh) de Realeza - MG, e um de areia lavada, para elaboração da curva-padrão de adsorção. considerada como material inerte.

Os solos foram previamente caracterizados física e quimicamente (Tabela 1), no Laboratório de Análises Física e Química de Solo, do Departamento de Solos, da UFV, segundo metodologia da EMBRAPA (1979). Em seguida. os solos foram passados em peneiras de malha de 4 $\mathrm{mm}$, secos ao ar e colocados em vasos plásticos. com capacidade para $430 \mathrm{~cm}^{3}$, recobertos internamente com sacos de polietileno.

Foram avaliadas, tanto para metribuzin quanto para flumioxazin, para cada solo, 10 doses dos herbicidas (Tabela 2). utilizando-se o delineamento experimental inteiramente casualizado, com cinco repetições. A parcela experimental foi constituída por um vaso, onde foram semeadas seis sementes de pepino (Cucumis 
sativus L.) Caipira 'AG 221', a $1,5 \mathrm{~cm}$ de profundidade. As doses aplicadas e a quantidade de solo adicionadas nos vasos variaram em função do tipo de solo.

Para aplicação dos herbicidas foi utilizado um pulverizador costal pressurizado com $\mathrm{CO}_{2}$ provido de barra de $0.5 \mathrm{~m}$ contendo dois bicos do tipo leque da série 80.03. A pressão foi de 3,0 $\mathrm{kgf} / \mathrm{cm}^{2}$, sendo o pulverizador calibrado para se obter vazão de 200 1/ha.

TABELA 1. Resultado das análises fisicas (análise granulométrica, umidade residual (Ure), densidade do solo (Dp), densidade da partícula (Dr) e porosidade (P)) e química dos solos Podzólico Vermelho-Amarelo câmbico fase terraço (PVc), Latossolo Roxo distrófico (LRd). Latossolo Vermelho-Amarelo textura média (LVm) e Latossolo Vermelho-Amarelo húmico (LVh).

\begin{tabular}{|c|c|c|c|c|}
\hline Análise granulométrica & $\mathrm{PVc}$ & LRd & $\mathrm{LVm}$ & LVh \\
\hline $\begin{array}{l}\text { Areia grossa }(\%) \\
\text { Areia fina }(\%) \\
\text { Silte }(\%) \\
\text { Argila }(\%) \\
\text { Ure }(\%) \\
\text { Dp }\left(\mathrm{g} / \mathrm{cm}^{3}\right) \\
\text { Dr }\left(\mathrm{g} / \mathrm{cm}^{3}\right) \\
\text { P }(\%) \\
\end{array}$ & $\begin{array}{c}34 \\
18 \\
06 \\
42 \\
2.77 \\
1.04 \\
2.53 \\
58.89 \\
\end{array}$ & $\begin{array}{c}13 \\
13 \\
16 \\
58 \\
8.11 \\
1.13 \\
3.03 \\
62.70 \\
\end{array}$ & $\begin{array}{c}28 \\
54 \\
01 \\
17 \\
0.40 \\
1.45 \\
2.60 \\
44.23 \\
\end{array}$ & $\begin{array}{c}12 \\
10 \\
09 \\
69 \\
2.57 \\
1,11 \\
2,56 \\
56,67 \\
\end{array}$ \\
\hline \multicolumn{5}{|l|}{ Análise quimica } \\
\hline $\begin{array}{l}\text { Carbono orgânico }(\mathrm{g} / \mathrm{kg}) \\
\mathrm{pH} \mathrm{H} \mathrm{H}_{2} \mathrm{O}(1: 2.5) \\
\mathrm{P}\left(\mathrm{mg} / \mathrm{dm}^{3}\right) \\
\mathrm{K}\left(\mathrm{mg} / \mathrm{dm}^{3}\right) \\
\mathrm{Al}\left(\mathrm{cmol}_{\mathrm{d}} / \mathrm{dm}^{3}\right) \\
\mathrm{Ca}\left(\mathrm{cmol}_{\mathrm{c}} / \mathrm{dm}^{3}\right) \\
\mathrm{Mg}\left(\mathrm{cmol}_{l} / \mathrm{dm}^{3}\right) \\
\mathrm{H}+\mathrm{Al}\left(\mathrm{cmol}_{/} / \mathrm{dm}^{3}\right) \\
\mathrm{SB}\left(\mathrm{cmol}_{\mathrm{c}} / \mathrm{dm}^{3}\right) \\
\text { CTC efetiva }\left(\mathrm{cmol}_{\mathrm{c}} / \mathrm{dm}^{3}\right) \\
\text { CTC total }\left(\mathrm{cmol}_{/} / \mathrm{dm}^{3}\right)\end{array}$ & $\begin{array}{c}30.4 \\
5.4 \\
2.9 \\
59 \\
0.0 \\
5.3 \\
0.9 \\
5.4 \\
6.28 \\
6.28 \\
11.68 \\
\end{array}$ & $\begin{array}{c}23.8 \\
4.8 \\
30.3 \\
195 \\
1.3 \\
0.9 \\
0.3 \\
9.0 \\
1.68 \\
2.98 \\
10.68 \\
\end{array}$ & $\begin{array}{c}5.1 \\
5.6 \\
0.3 \\
32 \\
0.0 \\
1.9 \\
0.6 \\
2.4 \\
2.57 \\
2.57 \\
4.97 \\
\end{array}$ & $\begin{array}{c}39.0 \\
6.4 \\
3.0 \\
20 \\
0.0 \\
4.2 \\
0.8 \\
4.8 \\
4.97 \\
4.97 \\
9.75 \\
\end{array}$ \\
\hline
\end{tabular}

TABELA 2. Doses (g/ha) de flumioxazin (flum) e de metribuzin (metr) aplicadas nos diferentes substratos, em casa-de-vegetação, UFV, MG.

\begin{tabular}{|c|c|c|c|c|c|c|c|c|c|}
\hline \multicolumn{10}{|c|}{ Substratos } \\
\hline \multicolumn{2}{|c|}{ PVc } & \multicolumn{2}{|c|}{ LRd } & \multicolumn{2}{|c|}{$\mathrm{LVm}$} & \multicolumn{2}{|c|}{ LVh } & \multicolumn{2}{|c|}{ Arcia } \\
\hline Flum & Metr & Flum & Metr & Flum & Metr & Flum & Metr & Flum & Metr \\
\hline \multicolumn{10}{|c|}{ Doses Aplicadas (g/ha) } \\
\hline 0 & 0 & 0 & 0 & 0 & 0 & 0 & 0 & 0 & () \\
\hline 5 & 30 & 2.5 & 5 & I & 2 & 10 & 40 & 1.5 & 4 \\
\hline 10 & 40 & 5.0 & 10 & 2 & 4 & 20 & 50 & 3,0 & 4 \\
\hline 15 & 50 & 7.5 & 15 & 3 & 6 & 30 & 60 & 4.5 & 6 \\
\hline 20 & 60 & 10 & 20 & 4 & 8 & 40 & 70 & 6.0 & 8 \\
\hline 25 & 70 & 12.5 & 25 & 5 & 10 & 50 & 80 & 7.5 & 10 \\
\hline 30 & 80 & 15 & 30 & 6 & 12 & 60 & 90 & 9.0 & 12 \\
\hline 40 & 90 & 17.5 & 35 & 7 & 14 & 70 & 100 & 10.5 & 14 \\
\hline 50 & 100 & 20 & 40 & 8 & 16 & 80 & 110 & 12 & 16 \\
\hline 60 & 110 & 22.5 & 45 & 9 & 18 & - & - & 13.5 & 18 \\
\hline
\end{tabular}


Imedia ta mente após a aplicação dos herbicidas, nos diferentes tratamentos, simulou-se, durante nove minutos, uma chuva de $12 \mathrm{~mm}$ nos vasos com solo, e de $8 \mathrm{~mm}$ nos vasos com areia, por se is minutos. Seis horas após a primeira irrigação e três vezes ao dia, para repor a água evapotranspirada, completou-se o volume de água, utilizando-se o simulador de chuva, a fim de que cada substrato atingisse $80 \%$ da capacidade de campo.

O teor de umidade de cada tipo de solo, foi previamente definido através de curva característica e mantido até a coleta das plantas, permitindo, assim, o máximo desenvolvimento da pl anta-teste e a maior disponibilidade dos herbicidas na solução do solo.

A coleta do experimento foi feita aos 18 dias após a semeadura, sendo avaliada a biomassa fresca da planta inteira de pepino, a qual foi dividida pelo número de plantas de cada vaso, obtendo-se a biomassa média por planta e por unid ad e experimental. Com esses valores, realizou-se a análise de variância, verificando-se o efeito de doses. Para os efeitos significativos, foi realizada análise de regressão. Para a escolha entre os mode los li near, quad rático e cúbico foi considerada a significância do teste $F$, maior $R^{2}$ e lógica biológica.

Determinou-se, para cada solo, a concentração de herbicida que proporcionou $50 \%$ de inibição no acúmulo de bio massa fresca da planta inteira, ou seja, o I.

Utilizando-se a metodologia proposta por Souza (1994), calculou-se a Relação de Adsorção (RA):

$$
R A=\frac{I_{50} \text { solo }-I_{50} \text { areia }}{I_{50} \text { areia }},
$$

avaliando-se quantas vezes a concentração necessária, para inibir 50\% do desenvolvimento da planta-teste, é adsorvida em cada tipo de solo.

\section{RESULTADOS E DISCUSSÃO}

Na Tabela 3 encontram-se as equações ajustadas para a biomassa fresca de plantas de 40 pe pi no e se us res pectivos coeficientes de determinação, em função das doses de flumioxazin e metribuzin, aplicadas em cada tipo de substrato. As doses dos herbicidas flumioxazin e metribuzin, que causaram $50 \%$ de inibição da produção de biomassa fresca de plantas de pepino ( $\left.\mathrm{I}_{50}\right)$, calculadas a partir das curvas ajustadas, e os respectivos valores de relação de adsorção (RA). estão apresentados na Tabela 4. O modelo linear foi o que melhor se ajustou à queda da biomassa das plantas de pepino, em função do aumento das doses de flumioxazin.

A quantidade requerida de metribuzin para inibir 50\% do desenvolvimento da planta-teste, em areia lavada, foi quase seis vezes maior que a necessária para flumioxazin, conforme verifica-se na Tabela 4. Isso demonstra que a atividade do flumioxazin foi maior que a do metribuzin, sendo, por is so. recomend ado $60 \mathrm{~g} / \mathrm{ha}$, do primeiro produto, em comparação a $480 \mathrm{~g} / \mathrm{ha}$ de metribuzin (Rodrigues \& Almeida, 1995).

Qua nto à capacidade ads or tiva dos diferentes tipos de solos em relação ao flumioxazin, verificou-se que no solo LVh, houve resposta linear negativa para biomassa fresca de pepino com o aumento das doses do flumioxazin. Esta elevada capacidade adsortiva do LVh, quanto ao flumioxazin (Tabela 4), pode ser atribuida aos ele vados teores de carbono orgânico $(\mathrm{CO})$ e de argila, existentes neste solo (Tabela 1). Segundo Klingman et al. (1975), solos com quantidades de matéria orgânica e argila elevadas, devido as suas maiores capacidades de adsorção, necessitam que doses maiores de herbicidas sejam aplicadas para controlar eficientemente as plantas daninhas.

No solo PVc, o aumento da dose de flumioxazin também proporcionou redução linear, porém menos intensa que no $\mathrm{LVh}$, no acúmulo de biomassa fresca de pepino. Como este solo é constituído por $52 \%$ de areia e apresenta aproximadamente $23 \%$ menos $\mathrm{CO}$ que o solo LVh, o seu poder adsortivo é menor, o que foi demonstrado pelos valores de $\mathrm{I}_{50}$ e RA (Tabela 4). Estudos realizados em Viçosa, por Carvalho Filho (1989), mostraram que a maior proporção da fração argila do solo PVc é de caulinita, seguida

Planta Daninha, v. 16, n. 1, 1998. 
de goetita, em menores teores. Segundo esse autor, a argila deste solo apresenta baixo valor de CTC, e é caracterizada por um capeamento de suas superficies pelo óxido de ferro livre, bloqueando, assim, os lugares de troca, o que explica, em parte, sua menor capacidade adsortiva, em relação ao solo LVh.

Os mais altos valores de $\mathrm{I}_{50}$, observados nos solos PVc e LVh, devem-se aos seus maiores teores de CO. A menor atividade do flumioxazin, observada no LRd, em comparação com PVc e LVh, pode ser atribuída ao baixo teor de $\mathrm{CO}$ e à qualidade da sua argila.

No solo LVm, o flumioxazin apresentou valores de $\mathrm{I}_{50}$ e RA inferiores aos de todos os outros solos estudados. A menor atividade se deve ao menor teor de $\mathrm{CO}$ e ao elevado teor de areia (Tabela 1).

A maior inativação do metribu zin ocorreu no solo de maior CTC (Tabela 4). Além de maior CTC, o solo PVc possui baixo a médio valor de pH. Estudos têm demonstrado que a elevação da CTC (Talbert \& Fletchall, 1965 e Sharom \& Stephenson, 1976), o decréscimo no pH (Peter \& Weber, 1985 e Harper, 1988) e a quantidade de argila e matéria orgânica do solo (Savage, 1976 e Peter \& Weber, 1985) aumentaram a adsorção do metribuzin pelo solo. Esse efeito do baixo valor de $\mathrm{pH}$ no solo PVc na adsorção do metribuzin, possivelmente, deve-se à protonação do grupo amina da molécula e à subsequente adsorção pelos colóide s do solo (Ladlie et al., 1976).

TABELA 3. Equações ajustadas para biomassa fresca de plantas de pepino submetidas a doses de flumioxazin e metribuzin, em diferentes substratos.

\begin{tabular}{|c|c|c|}
\hline Tipos de substratos & flumioxazin & metribuzin \\
\hline $\mathrm{PVc}$ & $\begin{array}{c}\wedge \\
y=3,6278-0,03119 . D \\
r^{2}=0,85\end{array}$ & $\begin{array}{c}\hat{A}=3,06691-0,0008422 . D- \\
0,00023588 . D^{2} \quad r^{2}=0,89\end{array}$ \\
\hline LVh & $\hat{y}=2,00588-0,01149 . D \quad r^{2}=0,95$ & $\begin{array}{c}\hat{y}=2,26355-0,01714 . D \\
r^{2}=0.93\end{array}$ \\
\hline LRd & $\begin{array}{c}\hat{y}=10,1152-0,1557, D \\
\hat{r}=0,78\end{array}$ & $\begin{array}{c}\hat{y}=3,12877-0,07364 . D \\
r^{2}=0,94\end{array}$ \\
\hline $\mathrm{LVm}$ & $\hat{\mathrm{y}}=3,28187-0,20936 . \mathrm{D} \mathrm{r} \mathrm{r}^{2}=0,89$ & $\hat{\mathrm{y}}=2,4096-0,074444 . D r^{2}=0,72$ \\
\hline Areia & $\hat{y}=2,2068-0,5046 . D$ & $\begin{array}{c}\hat{y}=2,23653-0,09034, D \\
r^{2}=0,89\end{array}$ \\
\hline
\end{tabular}

TABELA 4. Dose de flumioxazin ou de metribuzin que proporcionaram $50 \%$ de inibição ( $\left.\mathrm{I}_{50}\right)$ no acúmulo de biomassa fresca da planta inteira de pepino, e a respectiva relação de adsorção (RA), para os diferentes substratos.

\begin{tabular}{|c|c|c|c|c|}
\hline \multirow{2}{*}{$\begin{array}{c}\text { Tipos de } \\
\text { substratos }\end{array}$} & \multicolumn{2}{|c|}{$\mathrm{I}_{50}(\mathrm{~g} / \mathrm{ha})$} & \multicolumn{2}{|c|}{ RA } \\
\hline & flumioxazin & metribuzin & flumioxazin & metribuzin \\
\hline PVc & 58.16 & 81,00 & 25,56 & 5,54 \\
\hline LRd & 32.48 & 21.24 & 13,83 & 0,72 \\
\hline $\mathrm{LVm}$ & 7,84 & 16,18 & 2,58 & 0,31 \\
\hline LVh & 87,29 & 66,03 & 38,86 & 4,33 \\
\hline Areia & 2,19 & 12,38 & - & - \\
\hline
\end{tabular}


A maior atividade do metribuzin no solo LRd (Tabela 4) deve-se aos óxidos de ferro nele presentes. Maior adsorção do metribuzin ocorre pelos lugares hidrofilicos das áreas de superficie específica do solo (Peter \& Weber, 1985). Mais de 84\% da CTC total desse solo advém de $\mathrm{H}+\mathrm{Al}$, mostrando que a maioria de suas cargas provém de lugares dependentes de $\mathrm{pH}$.

Ainda para metribuzin, o solo $\mathrm{LVh}$ apresentou menor valor de RA, em relação ao solo PVc (Tabela 4), mesmo apresentando maior conteúdo de $\mathrm{CO}$ e argila (Tabela 1). Esses resultados são confirmados por Savage (1976), o qual verificou que a adição de matéria orgânica e argilas ao solo aumentou a adsorção do metribuzin, mas uma queda nessa adsorção foi observada quando níveis elevados de argila e matéria orgânica foram adicionados ao solo. Segundo esse autor, tal fato ocorreu devido à interação entre argila e matéria orgânica, associada à competição pelos lugares de adsorção. Segundo Rahman \& Matthews (1979), a matéria orgânica apresenta maior efeito na fitotoxicidade das striazinas com menor solubilidade em água do que as s-triazinas com alta solubilidade em água. A $\mathrm{p}_{\text {ior atividade do }}$ metribuzin no solo LVh, deve-se também ao alto valor de $\mathrm{pH}$ deste solo, conforme discutido anteriormente.

Pelas caracteristicas fisico-quimicas dos solos estudados, verificou-se que CO e argila, CTC e pH foram decisivos na expressão dos valores de RA para metribuzin, enquanto para flumioxazin $\mathrm{CO}$ e argila mostraram-se mais importantes.

Uma das possíveis causas da menor atividade no solo do flumioxazin, em relação ao metribuzin, entre outros fatores, é a sua menor solubilidade em água (0,6-1,2 ppm e 1.200 ppm, respectivamente). Isto pode ser explicado por diversos autores (Ladlie et al. 1976; Savage, 1976; Peter \& Weber, 1985: Shea, 1985: Graveel \& Turco, 1994 e Roowell, 1994), que correlacionaram a baixa solubilidade de herbicidas com altos valores de adsorção e. principalmente, com a matéria orgânica do solo.
No entanto, conclui-se que as doses dos herbicidas flumioxazin e metribuzin devem ser recomendadas em função do tipo de solo.

A recomendação de doses de flumioxazin e metribuzin para solos com altos teores de $\mathrm{CO}$ e argila deverá ser superior às doses recomendadas para solos com menores teores de $\mathrm{CO}$ e argila.

\section{LITERATURA CITADA}

BAILEY, C. W. \& WHITE, J. L. Factors influencing the adsorption and movement of pesticides in soil. In: Residue review the triazine herbicides, $v, 32$. New York. Springer Verlag. 1970. p. 29-92.

CARVALHO FILHO. A de Caracterizações mineralógicas, química e física de solos de duas unidades de paisagem do Planalto de Viçosa, MG. Viçosa - MG, UFV. 1989 Tese de Mestrado

DEUBER, R. Métodos de controle das plantas daninhas. In: Ciências das plantas daninhas. Jaboticabal: FUNEP, 1992. $p$ 109-148.

EMBRAPA (EMPRESA BRASILEIA DE PESQUISA AGROPECUÁRIA) - Serviço Nacional de Levantamento e Conservação de Solos. Manual de método de análise de solo. Rio de Janeiro. EMBRAPA-SNLCS 1979. n.p

GRAVEEL. J. G.: TURCO. R. F. Factors affecting mobility of pesticides in soil. In: Intensive course on the activity, selectivity, behavior, and fate of herbicides in plants and soils, 1994. West Lafayette, Indiana. USA. Herbicide action. West Lafayette: Purdue University. Departments of Horticulture. Agronomy. Botany and Plant Pathology, and Foresty and Natural Resources. 1994. p. $464-507$ 
HARPER, S. S. Sorption of metribuzin in surface and subsurface soils of the Mississipi Delta region. Weed Sci. v. $36 . n^{\circ}$ 1. p. 84-89, 1988.

KLINGMAN, G. C.; ASHTON, F. M.; NOORDHOFF, L. J. Weed Sci: principles and pratices. New York: John Wiley. 1975. $431 \mathrm{p}$.

LADLIE, J. S.; MEGGITT, W. F.; PENNER, D Effect of soil $\mathrm{pH}$ on microbial degradation. adsorption, and mobility of metribuzin. Weed Sci. v. 24, n. 5, p. 477-48 1, 1976

PETER, C. J. \& WEBER, J. B. Adsorption, mobility and efficacy of metribuzin as influenced by soil properties. Weed Sci. v. 33 , n.6, p. 868-873, 1985

RAHMAN. A. \& MATTHEWS. L. J. Effect of soil organic matter on the phytotoxicity of thirteen s-triazine herbicides. Weed Sci. v 27, n. 2, p.158-161. 1979

RODRIGUES, B. N. \& ALMEIDA, F. S. de (in memorian). Guia de Herbicidas - 3 ed. Londrina, PR. Brasil. 1995, 675 p.

RO0WELL, D. L. Pesticides and metals. In: Soil science: methods and applications. London, Longman Scientist Technical, 1994. 350 p.

SAVAGE, K. E. Adsorption and mobility of metribuzin in soil. Weed Sci. v. 24, n. I, p. 525-528, 1976.

SHAROM, M. S. \& STEPHENSON, G. R Behavior and fate of metribuzin in eight Ontario soils. Weed Sci. v. 24, n.2, p. 153160. 1976

SHEA. P. J. Detoxification of herbicide residues in soil. Weed Sci. v. 33, supp. 2, p. 33-41. 1985.

SOUZA, A. P. de, Atividade de oxyfluorfen, 2,4D e glifosate, em solos de diferentes texturas na presença e na ausência de composto orgânico. Viçosa - MG, UFV. 1994.71 p. (Tese de Mestrado).

STEVENSON. F.J. Organic matter reactions involving pesticides in soil. In: Humus chemistry, genisis, composition, reactions New York. Interscience, 1982. p. 428-430

TALBERT, R. E. \& FLETCHALL. O.H. Tho adsorption of some s-triazines in soils Weeds, v. 13, n. 1. p. 46-52, 1965. 